\title{
Determinants of Bumetanide Response in the Dog: Effect of Probenecid
}

\author{
David E. Smith ${ }^{1,2}$ and Henry S. H. Lau ${ }^{1}$
}

Received July 13, 1982-Final November 10, 1982

\begin{abstract}
The pharmacokinetics and pharmacodynamics of intravenous bumetanide $(0.250 \mathrm{mg} / \mathrm{kg})$, alone (treatment I) and after probenecid pretreatment (treatment II), were studied in four mongrel dogs. Lactated Ringer's solution was administered by vein throughout both treatments at a flow rate of $2 \mathrm{ml} / \mathrm{min}$ to avoid fuid and electrolyte depletion. Bumetanide and probenecid concentrations were analyzed by HPLC, sodium by Aame photometry, and creatinine by colorimetry. Although the probenecid markedly reduced the plasma and renal clearances of bumetanide, as well as the fraction excreted unchanged in the urine, there was no significant difference between treatments I and II in the 4-hr natriuretic and diuretic responses. However, analysis of the dose-response curves between treatments $I$ and $I I$ showed that sodium excretion was better correlated with bumetanide urinary excretion rate than with plasma concentration. The reasons for a poor correlation between treatments during the early time periods are discussed.
\end{abstract}

KEY WORDS: bumetanide; probenecid; pharmacokinetics; pharmacodynamics; doseresponse relationship.

\section{INTRODUCTION}

Bumetanide (3-n-butylamino-4-phenoxy-5-sulfamoylbenzoic acid) is a new high-ceiling diuretic which is $40-60$ times more potent than furosemide on a molecular weight basis $(1-3)$. The site of bumetanide action appears to be the thick ascending limb of the loop of Henle, where it inhibits solute reabsorption, although inhibition of sodium transport in the proximal tubule also occurs to a lesser extent (4-7). In addition, bumetanide has been shown to exert its natriuretic and diuretic effect from the luminal surface of the nephron (6). Since bumetanide is highly bound to plasma proteins $(8,9)$, access to the kidney lumen is limited through glomerular filtration. However, as a weak organic acid, bumetanide can enter the tubular fluid at the pars recta of the proximal tubule via the nonspecific organic acid secretory pathway $(1,8)$.

\footnotetext{
${ }^{1}$ College of Pharmacy, The University of Michigan, Ann Arbor, Michigan 48109.

${ }^{2}$ Author to whom correspondence should be addressed.
} 
Probenecid is a weak organic acid that competes with bumetanide for active secretion into the kidney lumen $(1,8)$. This competition can change the dose-response relationship of bumetanide by modifying either the total amount of diuretic delivered to its active site or the time course of drug delivery. Previous studies (10-13) evaluating the effect of probenecid on the pharmacodynamics of bumetanide have been limited and conflicting. These discrepancies probably reflect the inability of previous investigators (10-13) to measure the concentrations of bumetanide in the plasma and urine, presumably for the lack of a sensitive and specific assay method. Therefore, a rapid, sensitive, and specific high-performance liquid chromatographic (HPLC) assay was developed in an attempt to clarify the role of probenecid in modifying bumetanide's dose-response relationship.

\section{EXPERIMENTAL PROCEDURE}

\section{Materials}

An aqueous solution dosage form of bumetanide (Hoffmann-La Roche, Nutley, N. J., lot A-29), with the aid of $0.4 \mathrm{~N} \mathrm{NaOH}$, was prepared immediately prior to use. Probenecid capsules (Merck Sharp and Dohme, West Point, Pa., lot D2520) were obtained commercially. Probenecid powder (Merck Sharp and Dohme, Rahway, N.J.) was used as received. All other chemicals and solvents were reagent grade or better, as previously reported (14).

\section{Methods}

Four male, mongrel, conditioned, unanesthetized dogs weighing 21.5$32.5 \mathrm{~kg}$ received $0.250 \mathrm{mg} / \mathrm{kg}$ of bumetanide alone (treatment I) and $0.250 \mathrm{mg} / \mathrm{kg}$ of bumetanide after probenecid pretreatment (treatment II). Each dog fasted the night before and throughout the entire study period. Bumetanide was intravenously infused (Harvard Compact Infusion Pump, Harvard Apparatus Co., South Natick, Mass.) over a 3-min period with the beginning of the infusion being considered as time zero. A $1.0 \mathrm{~g}$ dose of probenecid ( 2 tablets $\times 0.5 \mathrm{~g} /$ tablet $)$ was ingested the night before $(11: 00-11: 30$ p.m. $)$ and on the study day $(60 \mathrm{~min}$ prior to bumetanide administration). An interval of at least one week elapsed between studies and identical lots for each drug were used throughout.

Heparinized scalp vein needles (E-Z Set-PRN Intermittent Infusion Set, The Deseret Co., Sandy, Utah) were placed in each foreleg of the dogs, one for administration of bumetanide and replacement fluids and one for obtaining blood samples. Blood samples $(3 \mathrm{ml})$ were collected just prior to bumetanide dosing (blank) and at $3,5,10,20,30,45,60,80$, 
$100,120,150,180,210$, and $240 \mathrm{~min}$. Voided urine was collected via an indwelling bladder catheter (Swan-Ganz Flow-Directed Monitoring Catheter, Model 93-111-7F, American Edwards Laboratories, Santa Ana, Calif.) just prior to bumetanide dosing (blank) and at 20,40, 80, 120, 180, and $240 \mathrm{~min}$. The bladder was flushed with $2 \times 5 \mathrm{ml}$ of air at the end of each urine collection to insure a complete catch. Lactated Ringer's solution was administered by vein throughout the entire study period for both treatments I and II at a flow rate of $2 \mathrm{ml} / \mathrm{min}$ to avoid fluid and electrolyte depletion. All 4-hr plasma samples showed normal sodium concentrations.

\section{Assays}

Plasma and urine samples containing bumetanide, with and without probenecid pretreatment, were analyzed by an HPLC method as recently reported (14). Probenecid concentrations in plasma were simultaneously determined with bumetanide concentrations using the same sample preparation, instrumentation, and solvent system. However, both probenecid and the internal standard acetophenone were measured using ultraviolet detection $(254 \mathrm{~nm})$ and had retention times in plasma of 8.0 and $4.0 \mathrm{~min}$, respectively (Fig. 1). A representative standard curve of probenecidacetophenone peak height ratio over the probenecid plasma concentration

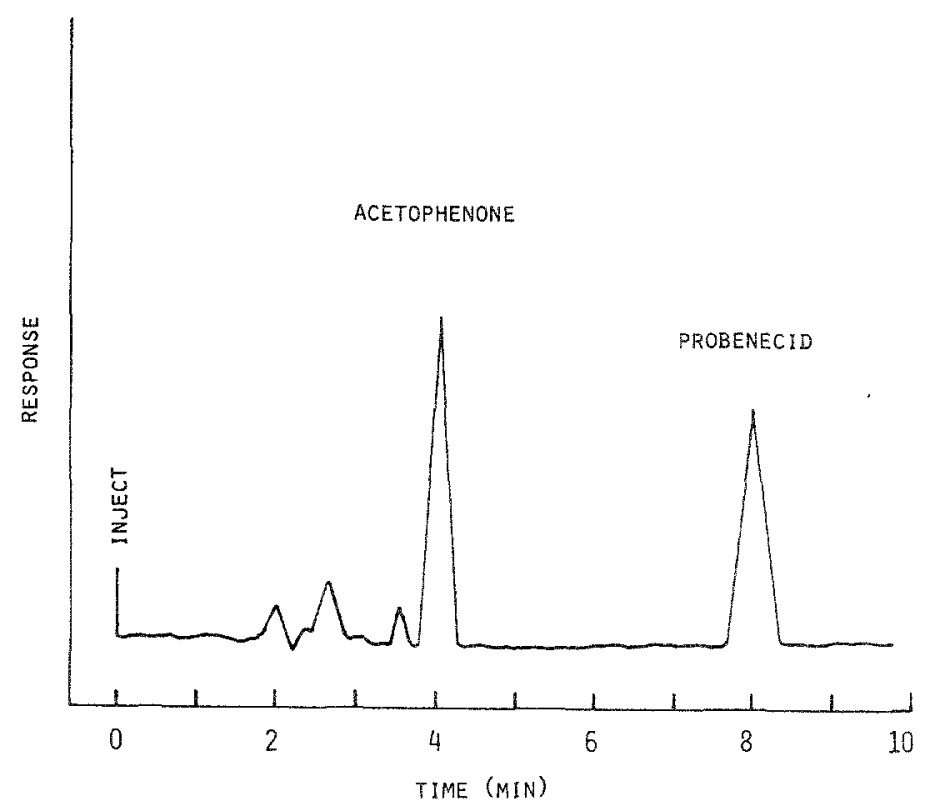

Fig. 1. Chromatogram for plasma spiked with probenecid and the internal standard, acetophenone, using ultraviolet detection (254 nm). 
range $50-750 \mu \mathrm{g} / \mathrm{ml}$ resulted in the following linear least-squares regression equation: $Y=0.003 X+0.011 ; r^{2}=0.999$. Plasma and urine samples were measured for sodium with a flame photometer (Model 455, Corning Medical and Scientific, Medfield, Mass.), and creatinine was determined colorimetrically using a commercial kit (Sigma Chemical Company, St. Louis, Mo.).

\section{Calculations}

Plasma concentration-time curves of bumetanide were fitted (equally weighted) to the general polyexponential equation for post constant-rate infusion data (15):

$$
C p=\sum_{i=1}^{n} Y i e^{-\lambda i t}
$$

In Eq. (1), $C p$ represents the plasma concentration at time $t, Y i$ is the coefficient of the $i$ th exponential term for post constant-rate intravenous infusion data, and $\lambda i$ is the exponent of the $i$ th exponential term. The values of the coefficients and exponential terms in Eq. (1) were obtained using a nonlinear least-squares regression program (personal communication, Dr. J. L. Fox, College of Pharmacy, The University of Michigan, Ann Arbor, Mich.) and a microcomputer (Apple II Plus Computer, Apple Computer Inc., Cupertino, Calif.). Initial estimates were obtained using the program RSTRIP (personal communication, Dr. J. L. Fox, College of Pharmacy, The University of Michigan, Ann Arbor, Mich.). The number of exponents $(n)$ needed for each data set were determined by the application of Akaike's information criterion (16). Since

$$
Y i=\sum_{i=1}^{n}\left(1-e^{\lambda i T}\right) C i /(-\lambda i T)
$$

where $T$ is the constant-rate infusion time and $\mathrm{Ci}$ is the coefficient of the $i$ th exponential term for bolus intravenous data, Eq. (1) can be rearranged to the corresponding equation (ref. 15):

$$
C p=\sum_{i=1}^{n}\left(1-e^{\lambda i T}\right) C i e^{-\lambda i t} /(-\lambda i T)
$$

Once the values of the coefficients and exponential terms in Eq. (1) are determined by computer fitting, the values of $C i$ in Eq. (3) can be calculated.

The following pharmacokinetic parameters were calculated using standard equations (refs. 15, 17):

$$
V_{1}=D / \sum_{i=1}^{n} C i
$$




$$
\begin{gathered}
V_{d s s}=D \sum_{i=1}^{n} C i / \lambda i^{2} /\left(\sum_{i=1}^{n} C i / \lambda i\right)^{2} \\
V_{\text {darea }}=D /\left(\lambda_{1} \sum_{i=1}^{n} C i / \lambda i\right) \\
C L_{p}=D / \sum_{i=1}^{n} C i / \lambda i \\
C L_{r}=A e^{\infty} / \sum_{i=1}^{n} C i / \lambda i \\
C L_{n r}=C L_{p}-C L_{r} \\
T_{1 / 2}=0.693 / \lambda_{1} \\
K_{10}=C L_{p} / V_{1} \\
f e=A e^{\infty} / D
\end{gathered}
$$

In Eqs. (4)-(12), $V_{1}$ is the volume of the central compartment; $V_{d s s}$ is the volume of distribution steady-state; $V_{\text {darea }}$ is that volume which, when multiplied by $C p$ in the log-linear phase, is equal to the amount of drug in the body; $D$ is the intravenous dose (equal to the product of the zero-order infusion rate and the length of infusion); $C_{1}$ and $\lambda_{1}$ are the coefficient and exponent, respectively, such that $\lambda_{1}$ is the smallest of the $\lambda i$ 's of the polyexponential equation; $C L_{p}$ is the total plasma clearance; $C L_{r}$ is the renal clearance; $C L_{n r}$ is the nonrenal clearance; $A e^{\infty}$ is the amount of unchanged drug recovered in the urine at time infinity; $T_{1 / 2}$ is the biologic half-life; $K_{10}$ is the first-order elimination rate constant from the central compartment; and $f e$ is the fraction of the available dose excreted unchanged in the urine. Creatinine clearance $\left(C L_{c r}\right)$ was calculated by dividing the urinary excretion rate of creatinine by its plasma concentration at the midpoint of the urine collection period.

Data throughout the study are expressed as the means $\pm S D$, unless otherwise indicated. Statistical differences between treatments I and II were determined by a paired $t$-test. Statistical differences within a specific treatment were determined by a single factor analysis of variance and Newman-Keuls multiple range test. A $p$ value $<0.05$ was considered to be significant.

\section{RESULTS}

Plasma concentrations of bumetanide over 4 hr were fitted to a biexponential equation for six data sets and to a triexponential equation for 
Table 1. Coefficients and Exponential Terms of Bumetanide Obtained Using Biexponential and Triexponential Equations

\begin{tabular}{cccccccc}
\hline & & \multicolumn{5}{c}{ Biexponential Equation } \\
\cline { 3 - 6 } Dog & Treatment $^{a}$ & $\begin{array}{c}C_{1} \\
(\mathrm{ng} / \mathrm{ml})\end{array}$ & $\begin{array}{c}C_{2} \\
(\mathrm{ng} / \mathrm{ml})\end{array}$ & $\begin{array}{c}\lambda_{1} \\
\left(\mathrm{~min}^{-1}\right)\end{array}$ & $\begin{array}{c}\lambda_{2} \\
\left(\mathrm{~min}^{-1}\right)\end{array}$ & $R^{2 b}$ & CORR $^{c}$ \\
\hline 1 & I & 120 & 1855 & 0.0095 & 0.1150 & 0.991 & 0.994 \\
& II & 309 & 1342 & 0.0099 & 0.1035 & 0.999 & 0.999 \\
2 & I & 119 & 1634 & 0.0102 & 0.1016 & 0.997 & 0.998 \\
& II & 1023 & 1245 & 0.0175 & 0.1319 & 0.999 & 0.999 \\
3 & I & 125 & 1978 & 0.0125 & 0.1166 & 0.996 & 0.997 \\
4 & I & 164 & 1187 & 0.0134 & 0.1265 & 0.995 & 0.996 \\
\hline
\end{tabular}

Triexponential Equation

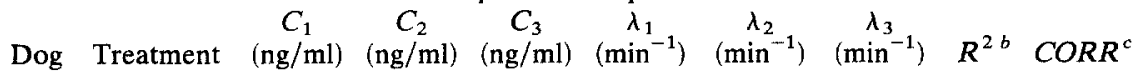

\begin{tabular}{rrrrrrrrrr}
\hline 3 & II & 406 & 1115 & 908 & 0.0125 & 0.0655 & 0.2786 & 0.999 & 0.999 \\
4 & II & 188 & 756 & 1937 & 0.0074 & 0.0415 & 0.1859 & 0.999 & 0.999 \\
\hline
\end{tabular}

${ }^{a}$ Treatment I represents bumetanide before probenecid pretreatment. Treatment II represents bumetanide after probenecid pretreatment.

${ }^{b} R^{2}=\left[\Sigma(\mathrm{Obs})^{2}-\Sigma(\mathrm{Dev})^{2}\right] / \Sigma(\mathrm{Obs})^{2}$.

'CORR represents the correlation between the calculated and observed plasma concentrations.

two data sets (Table I). The goodness of the fit, as determined by $R^{2}$ and CORR, was 0.991 or higher.

The pharmacokinetics of bumetanide alone (treatment I) and after probenecid pretreatment (treatment II), are presented in Table II. Total plasma clearance of bumetanide was significantly reduced after probenecid pretreatment $(9.65 \pm 1.32$ for treatment I vs $4.69 \pm 0.80 \mathrm{ml} / \mathrm{min} \cdot \mathrm{kg}$ for treatment II; $p<0.01$ ), resulting in significantly higher plasma concentrations of the diuretic, except for the first $5 \mathrm{~min}$ (Fig. 2). The change in total plasma clearance was due to the dramatic reduction (six-fold) in bumetanide renal clearance $(4.55 \pm 0.89$ for treatment I vs $0.759 \pm 0.154 \mathrm{ml} / \mathrm{min} \cdot \mathrm{kg}$ for treatment II; $p<0.005$ ) since the nonrenal clearance did not significantly differ between treatments. As a consequence of the reduced renal clearance, a significantly smaller fraction of the bumetanide dose was excreted unchanged in the urine in the presence of probenecid $(0.470 \pm 0.029$ for treatment I vs $0.164 \pm 0.032$ for treatment II; $p<0.002$ ). This reduction in the urinary excretion of bumetanide was observed to occur predominantly during the first 40 min following administration of the diuretic (Fig. 3). $T_{1 / 2}$ did not change after probenecid pretreatment although $K_{10}$ was significantly reduced. This demonstrates the unreliability of biologic halflife as an indicator of capacity-limited mechanisms since its value depends 


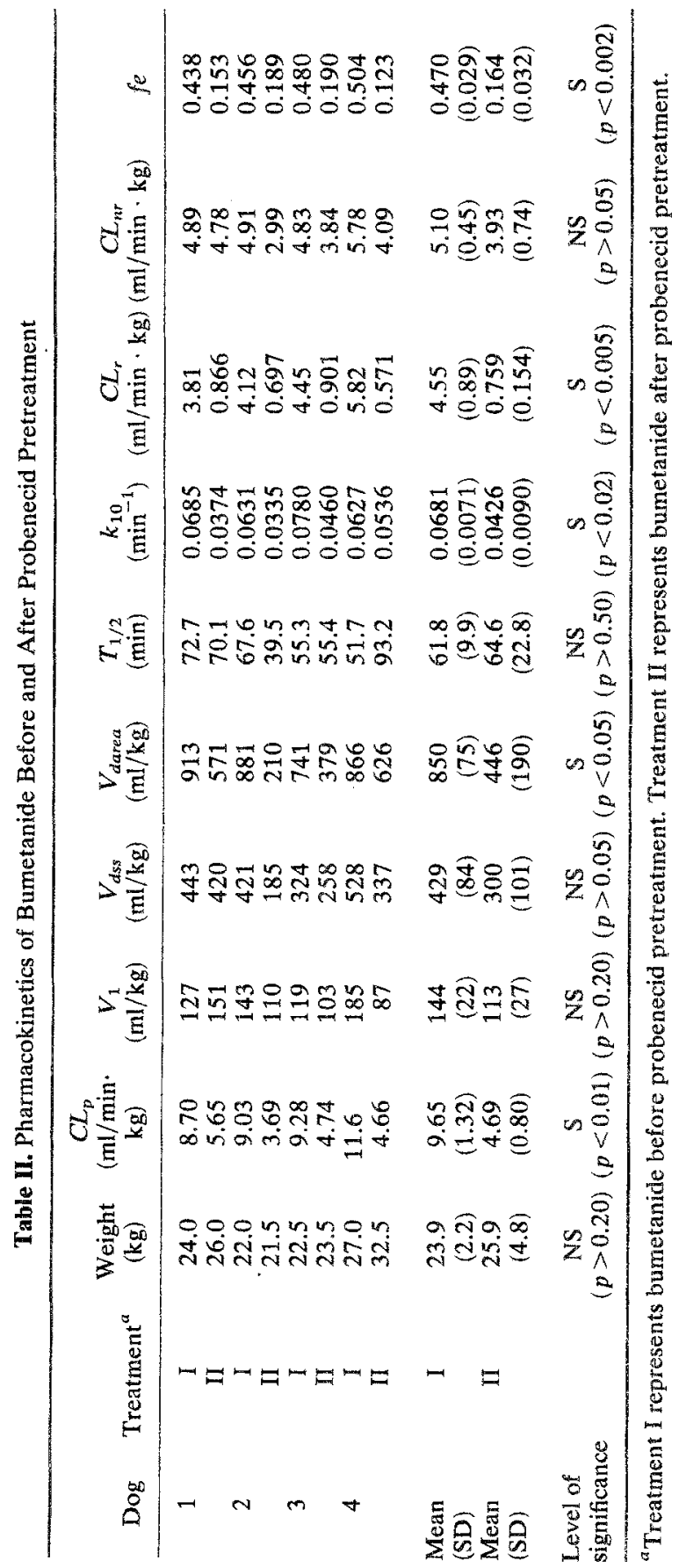




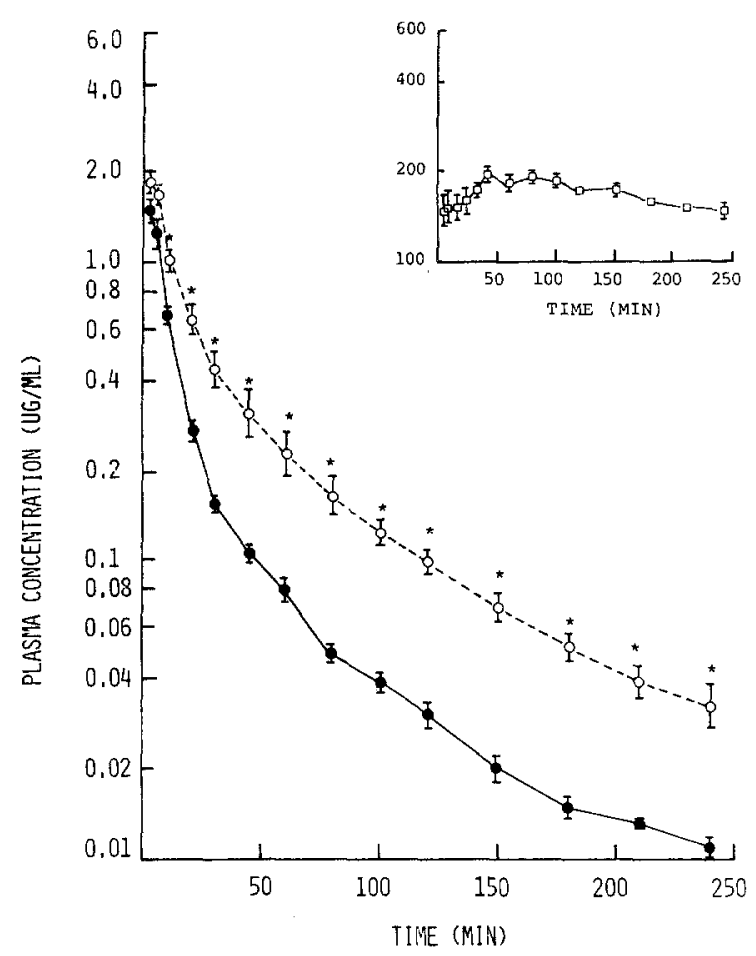

Fig. 2. Plasma concentration vs time plots of bumetanide alone (-), bumetanide after probenecid pretreatment $\left(\mathrm{O}-\mathrm{O}^{-}\right)$, and probenecid $(\square-\square)$. Data are expressed as the mean $\pm \operatorname{SEM}(n=4)$. Asterisks denote statistical differences between treatments I and II.

upon distributive as well as elimination characteristics of the drug. The $V_{\text {darea }}$ of bumetanide was significantly diminished by concomitant administration with probenecid while $V_{1}$ did not change. $V_{d s s}$ showed a $30 \%$ decrease after probenecid pretreatment. However, the change was not significant at the $95 \%$ confidence level and probably reflects the limited number of dogs studied $(0.10>p>0.05)$.

The effects of probenecid on bumetanide-induced diuresis and natriuresis are presented in Table III. Pharmacodynamic data are reported as electrolyte excretion rate and cumulative excretion (as opposed to fractional excretion) since sodium concentrations and creatinine clearances did not change between treatments $\left(C L_{c r}=2.49 \pm 0.24\right.$ for treatment I vs $2.20 \pm 0.50 \mathrm{ml} / \mathrm{min} \cdot \mathrm{kg}$ for treatment II; $p>0.20$ ). Probenecid pretreatment had no significant effect on the cumulative urine volume $(1060 \pm 77$ for treatment I vs $942 \pm 79 \mathrm{ml} / 4 \mathrm{hr}$ for treatment II; $p>0.20$ ), the cumula- 


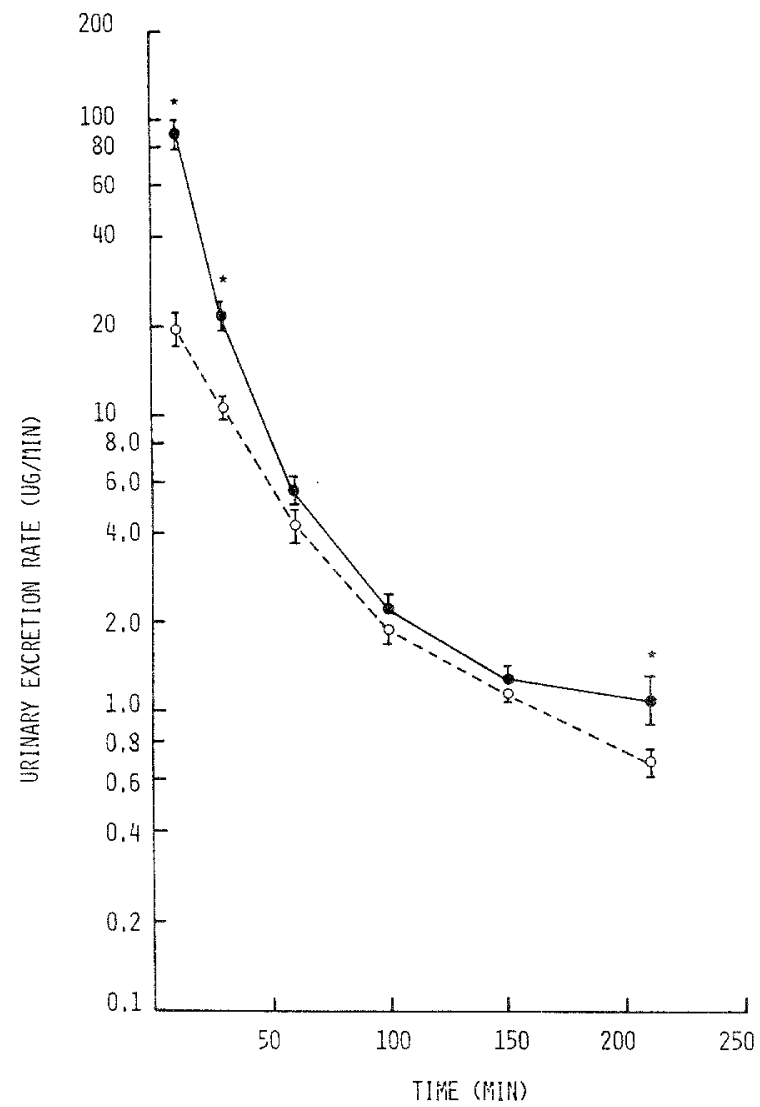

Fig. 3. Urinary excretion rate vs midpoint time plots of bumetanide alone (-) and bumetanide after probenecid pretreatment $(0$ - - O). Data are expressed as the mean $\pm \operatorname{SEM}(n=4)$. Asterisks denote statistical differences between treatments I and II.

tive sodium excretion $(121 \pm 17$ for treatment I vs $116 \pm 11 \mathrm{mEq} / 4 \mathrm{hr}$ for treatment II; $p>0.50$ ), or the time course of bumetanide-induced natriuresis (Fig. 4). In addition, Fig. 4 demonstrates that the maximal response to intravenous bumetanide occurred approximately $30 \mathrm{~min}$ after dosing, indicating a time lag for drug effect.

\section{DISCUSSION}

Experiments in animals and humans have demonstrated that the critical determinant with respect to furosemide's natriuretic and diuretic effect is 
Table III. Effects of Probenecid on Bumetanide Diuresis and Natriuresis

\begin{tabular}{crcc}
\hline Dog & Treatment $^{a}$ & $\begin{array}{c}\text { Urine volume } \\
\text { (ml/4 hr) }\end{array}$ & $\begin{array}{c}\text { Sodium excretion } \\
(\mathrm{mEq} / 4 \mathrm{hr})\end{array}$ \\
\hline 1 & I & 1131 & 123 \\
& II & 922 & 105 \\
2 & I & 1028 & 116 \\
& II & 984 & 123 \\
3 & I & 967 & 103 \\
4 & II & 1022 & 128 \\
& I & 1115 & 143 \\
Mean & II & 842 & 107 \\
(SD) & I & 1060 & 121 \\
Mean & II & $(77)$ & $(17)$ \\
(SD) & & 942 & 116 \\
& & $(79)$ & $(11)$ \\
Level of significance & & NS & NS \\
& & $(p>0.20)$ & $(p>0.50)$
\end{tabular}

${ }^{a}$ Treatment I represents bumetanide before probenecid pretreatment. Treatment II represents bumetanide after probenecid pretreatment.

the drug's luminal concentration or amount rather than its plasma concentration (18-22). The determinants of bumetanide response are less well understood although this new loop diuretic has a pharmacologic action similar to that of furosemide. Since previous investigators (10-13) did not measure the concentrations of bumetanide in plasma and urine, they were unable to establish a relationship between the pharmacodynamic response of bumetanide and concentrations of drug in a sampling compartment reflective of its site of action.

Friedman and Roch-Ramel (10) studied the renal hemodynamic and natriuretic effects of bumetanide and furosemide in cats. They reported that probenecid $(50 \mathrm{mg} / \mathrm{kg}$ i.v. $)$ failed to block the natriuretic effect of bumetanide $(0.025 \mu \mathrm{g} / \mathrm{min} \cdot \mathrm{kg})$ when infused into one renal artery but abolished the natriuretic action of furosemide $(1.0 \mu \mathrm{g} / \mathrm{min} \cdot \mathrm{kg})$. The authors (10) proposed two possible mechanisms to explain this finding: first, that the renal tubular transport of bumetanide was not interfered with by probenecid, in contrast to furosemide, due to a greater affinity of bumetanide for the transport system; and second, that bumetanide might reach its site of action through the peritubular membrane. However, Holland and Williamson (11) subsequently demonstrated in dogs that probenecid pretreatment $(50 \mathrm{mg} / \mathrm{kg}$ i.v. $)$ could significantly block the natriuretic and diuretic actions of bumetanide $(0.05 \mu \mathrm{g} / \mathrm{min} \cdot \mathrm{kg})$, presumably by inhibiting its tubular secretion. The reason for the discrepancy 


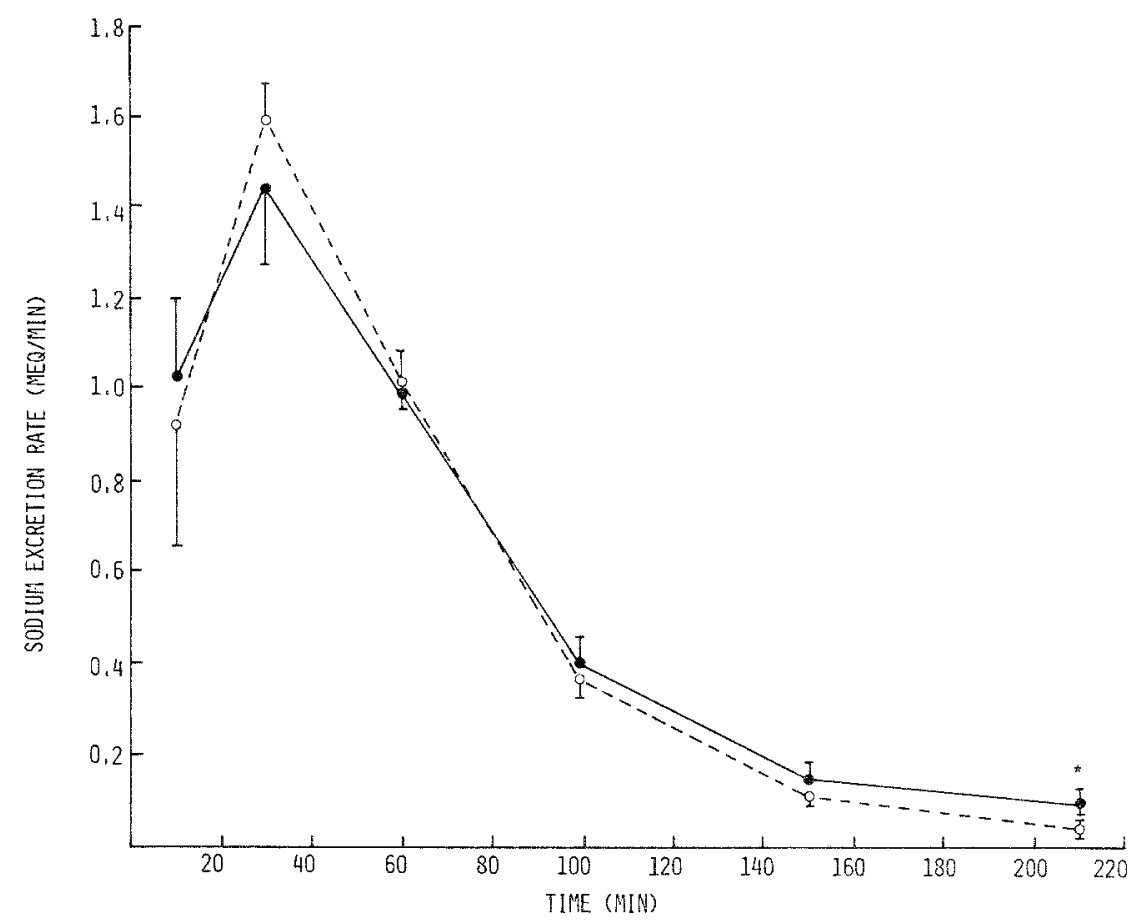

Fig. 4. Sodium excretion rate vs midpoint time plots of bumetanide alone bumetanide after probenecid pretreatment (O-- 0 ). Data are expressed as SEM $(n=4)$. Asterisks denote statistical differences between treatments I and II.

between these two studies $(10,11)$ is difficult to reconcile since their experimental designs were similar. Perhaps a species difference exists between the cat and dog with respect to their organic acid transport systems.

Conflicting reports have also appeared in the literature concerning the effect of probenecid on bumetanide response in healthy volunteers $(12,13)$. In 1975, Lant (12) reported that in two healthy subjects probenecid blocked a major part of the excretory responses evoked by $0.5 \mathrm{mg}$ intravenous bumetanide. However, the urinary responses to $3 \mathrm{mg}$ bumetanide were largely unaltered by this agent. Thus, it appears that at higher doses of bumetanide, a sufficient amount of drug can enter the tubular lumen in the presence of probenecid to produce an adequate natriuretic response. In contrast, Brater and Chennavasin (13) observed that in eight healthy subjects, probenecid affected neither the cumulative response nor time course of response to 0.5 and $1.0 \mathrm{mg}$ doses of bumetanide. These authors (13) suggest that probenecid does not affect the renal handling of 


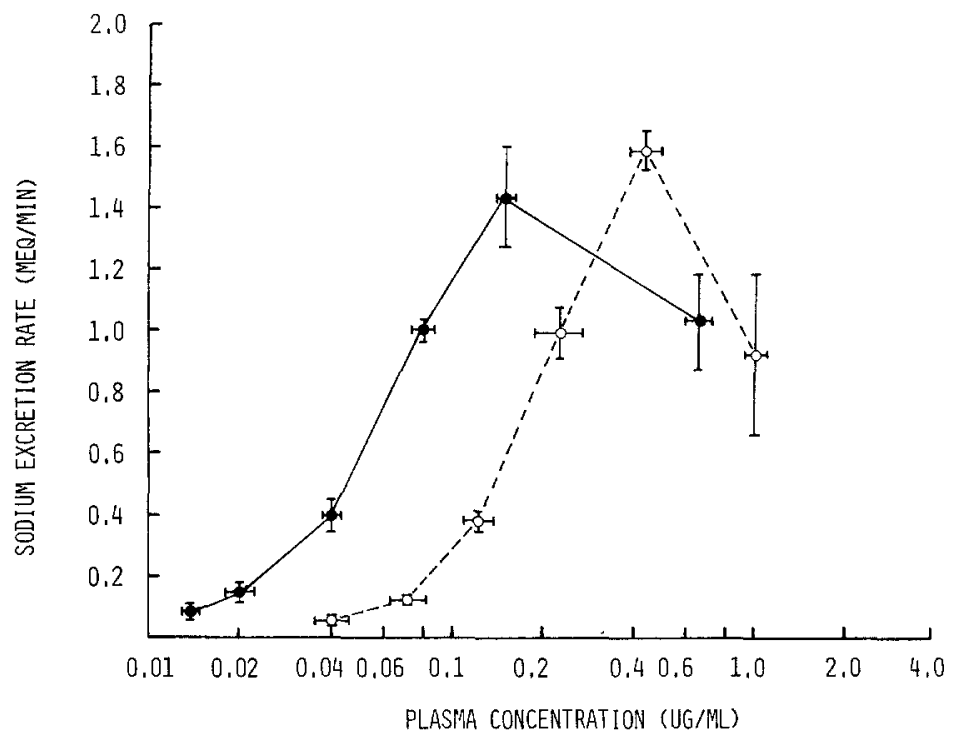

Fig. 5. Sodium excretion rate vs plasma concentration plots of bumetanide alone (-O) and bumetanide after probenecid pretreatment $(0 \ldots$.$) .$ Data are expressed as the mean $\pm \operatorname{SEM}(n=4)$,

bumetanide, although they acknowledge that confirmation of this hypothesis requires the measurement of bumetanide concentrations.

In the present study, we demonstrated that probenecid does inhibit the active secretion of bumetanide into the tubular fluid of dogs, as evidenced by the dramatic reduction in $C L_{r}$ and $f e$. Despite this inhibition, there were no significant differences between treatments I and II in the 4-hr natriuretic and diuretic responses. Therefore, the time course of bumetanide in plasma and urine was analyzed with respect to the natriuretic effect in an attempt to define the determinants of bumetanide response.

Figure 5 shows that probenecid caused a significant shift to the right in the relationship between sodium excretion rate and the logarithm of bumetanide plasma concentration over the entire dose-response curve. This effect is consistent with that of a competitive inhibitor. Furthermore, it suggests that higher plasma concentrations of bumetanide are required in the presence of probenecid to elicit a natriuretic response equivalent to that produced by lower plasma concentrations when probenecid is absent. In contrast, sodium excretion rate was found to be better correlated with the logarithm of bumetanide urinary excretion rate (Fig. 6). Still, poor correlations were observed between treatments I and II during the first $40 \mathrm{~min}$ after bumetanide administration (two highest values for urinary excretion rate in each treatment). This finding reflects the following two 


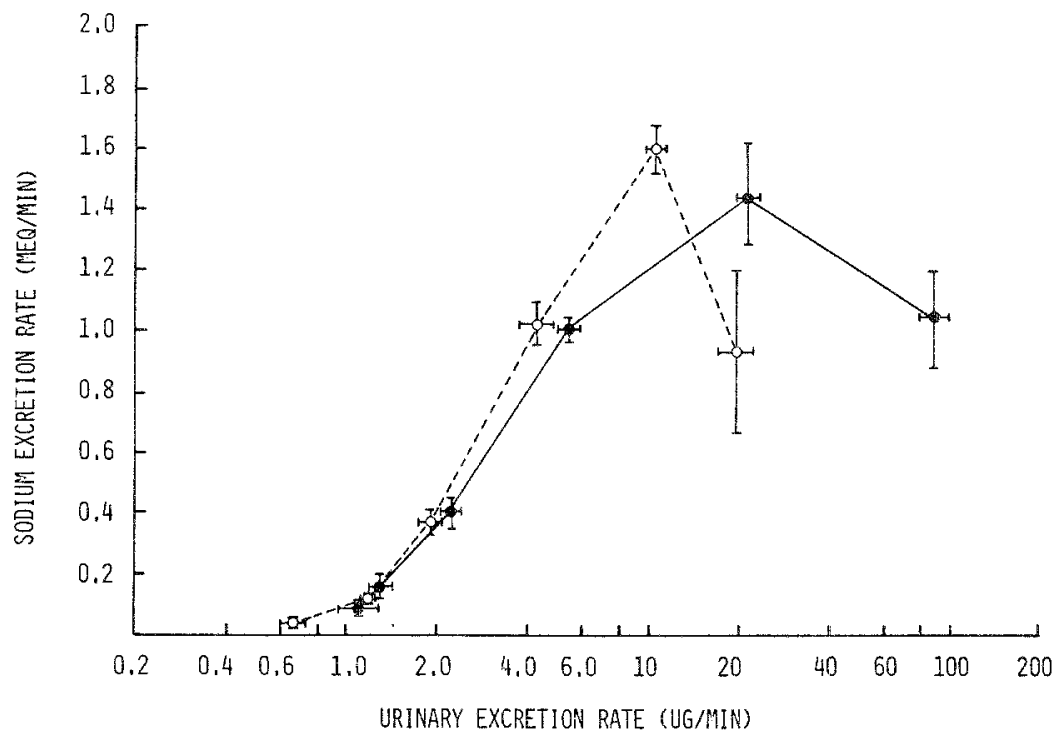

Fig. 6. Sodium excretion rate vs urinary excretion rate plots of bumetanide alone (-) and bumetanide after probenecid pretreatment $(\mathrm{O} \ldots \mathrm{O})$. Data are expressed as the mean $\pm \operatorname{SEM}(n=4)$.

complications which exist when analyzing the data. First, the present model does not accommodate the disequilibrium between the urine concentration and effect compartment (biophase) which occurs during the early distribution phase (23). Second, bumetanide demonstrates a nonlinear renal clear-ance during these early time periods for treatment I only (Fig. 7).

Although speculative, a mechanism consistent with this change in bumetanide renal clearance would be a reduction in tubular reabsorption at a location distal to the diuretic's site of action. This could account for the greater than proportional amount of unchanged drug being excreted into the urine during the first $40 \mathrm{~min}$ of treatment I without a concomitant increase in bumetanide response. The reason why a nonlinear renal clearance of bumetanide is not observed during treatment II is unclear. Perhaps probenecid interferes with the tubular reabsorption of bumetanide such that changes in urine flow and/or $\mathrm{pH}$ are not important in affecting the diuretic's renal clearance. A similar finding with respect to furosemide was recently reported by Green and Mirkin (24). They observed that in rats, urinary acidification produced a profound decrease in the renal clearance of furosemide compared to those animals with a more alkaline urine. However, the diuretic response did not differ among the animals despite the alteration in furosemide renal clearance. This implies that an equivalent amount of furosemide reaches the loop of Henle in both circumstances 


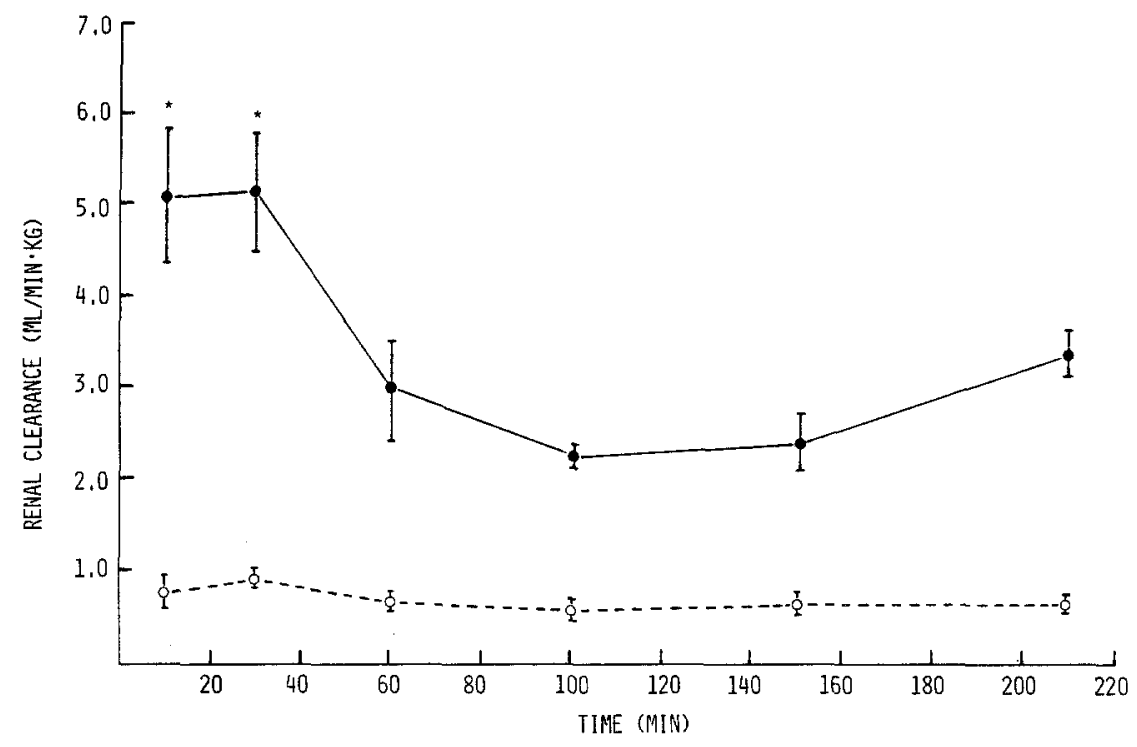

Fig. 7. Renal clearance vs midpoint time plots of bumetanide alone (- and bumetanide after probenecid pretreatment $\left(\mathrm{O}_{--} \mathrm{O}\right)$. Data are expressed as the mean \pm $\operatorname{SEM}(n=4)$. Asterisks denote statistical differences within a specific treatment.

$\left(\mathrm{HCl}\right.$ vs $\mathrm{NaHCO}_{3}$ treated rats). The authors (24) state that the most likely mechanism for a decreased renal clearance of furosemide is therefore tubular reabsorption from the acidified urine occurring in the distal tubule and collecting duct.

In conclusion, although probenecid caused marked changes in the pharmacokinetic parameters of bumetanide (decreased plasma and renal clearances, decreased $V_{\text {darea }}$ and $K_{10}$, and decreased fraction excreted unchanged in the urine), there was no significant difference between treatments I and II in the cumulative response or time course of response to the diuretic. However, analysis of the dose-response curves between treatments I and II showed that bumetanide urinary excretion rate was a better indicator of natriuresis and diuresis than was the plasma concentration. Furthermore, these results support the hypothesis that adequate luminal concentrations or amounts of bumetanide are a prerequisite for the drug's pharmacodynamic effects.

\section{ACKNOWLEDGMENTS}

This work was supported in part by a Biomedical Research Support Grant from the College of Pharmacy and a Faculty Research Rackham 
Grant, The University of Michigan. During the course of this work H.S.H. Lau was supported as an NIH Predoctoral Scholar on NIH Training Grant GM 07767-04. The authors are grateful to Dr. J. L. Fox for the availability of his computer programs and expertise.

\section{REFERENCES}

1. H. H. Frey. Pharmacology of bumetanide. Postgrad. Med. I. Suppl. 51:14-18 (1975).

2. U. B. Olsen. The pharmacology of bumetanide: a review. Acta Pharmacol. Toxicol. Suppl. III:3-29 (1977).

3. M. Cohen. Pharmacology of bumetanide. J. Clin. Pharmacol. 21:537-542 (1981).

4. E. Bourke, M. J. A. Asbury, S. O'Sullivan, and P. B. B. Gatenby. The sites of action of bumetanide in man. Eur. J. Pharmacol. 23:283-289 (1973).

5. S. Jayakumar and J. B. Puschett. Study of the sites and mechanisms of action of bumetanide in man. J. Pharmacol. Exp. Ther. 201:251-258 (1977).

6. M. Imai. Effect of bumetanide and furosemide on the thick ascending limb of Henle's loop of rabbits and rats perfused in vitro. Eur. J. Pharmacol. 41: 409-416 (1977).

7. S. G. Karlander, R. Henning, and $O$. Lundvall. Renal effects of bumetanide, a new saluretic agent. Eur. J. Clin. Pharmacol. 6:220-223 (1973).

8. E. H. Østergaard, M. P. Magnussen, C. K. Nielsen, E. Eilertsen, and H. H. Frey. Pharmacological properties of 3-n-butylamino-4-phenoxy-5-sulfamylbenzoic acid (bumetanide), a new potent diuretic. Arzneim.-Forsch. 22:66-72 (1972).

9. M. R. Cohen, E. Hinsch, R. Vergona, J. Ryan, S. J. Kolis, and M. A. Schwartz. A comparative diuretic and tissue distribution study of bumetanide and furosemide in the dog. J. Pharmacol. Exp. Ther. 197:697-702 (1976).

10. P. A. Friedman and F. Roch-Ramel. Hemodynamic and natriuretic effects of bumetanide and furosemide in the cat. J. Pharmacol. Exp. Ther. 203:82-91 (1977).

11. S. D. Holland and H. E. Williamson. Probenecid inhibition of bumetanide-induced natriuresis in the dog. Proc. Soc. Exp. Biol. Med. 161:299-302 (1979).

12. A. F. Lant. Effects of bumetanide on cation and anion transport. Postgrad. Med. I. Suppl. 51:35-42 (1975).

13. D. C. Brater and P. Chennavasin. Effect of probenecid on response to bumetanide in man. J. Clin. Pharmacol. 21: 311-315 (1981).

14. D. E. Smith. High-performance liquid chromatographic assay for bumetanide in plasma and urine. J. Pharm. Sci. 71: 520-523 (1982).

15. J. G. Wagner. Pharmacokinetic parameters estimated from intravenous data by uniform methods and some of their uses. J. Pharmacokin. Biopharm. 5:161-182 (1977).

16. K. Yamaoka, T. Nakagawa, and T. Uno. Application of Akaike's information criterion (AIC) in the evaluation of linear pharmacokinetic equations. I. Pharmacokin. Biopharm. 6:165-175 (1978).

17. J. G. Wagner. Fundamentals of Clinical Pharmacokinetics. Drug Intelligence Publications, Hamilton, Ill., 1975, pp. 38, 83, 349.

18. L. Z. Benet. Pharmacokinetics/pharmacodynamics of furosemide in man: a review $J$. Pharmacokin. Biopharm. 7:1-27 (1979).

19. P. Chennavasin, R. Seiwell, D. C. Brater, and W. M. M. Liang. Pharmacodynamic analysis of the furosemide-probenecid interaction in man. Kidney Int. 16:187-195 (1979).

20. D. E. Smith and L. Z. Benet. Relationship between urinary excretion rate, steady-state plasma levels and diuretic response of furosemide in the rat. Pharmacology 19:301-306 (1979).

21. D. E. Smith, W. L. Gee, D. C. Brater, E. T. Lin, and L. Z. Benet. Preliminary evaluation of furosemide-probenecid interaction in humans. J. Pharm. Sci. 69:571-575 (1980).

22. T. P. Green and B. L. Mirkin. Resistance of proteinuric rats to furosemide: urinary drug protein binding as determinant of drug effect. Life Sci. 26: 623-630 (1980). 
23. N. H. G. Holford and L. B. Sheiner. Understanding the dose-effect relationship: clinical application of pharmacokinetic-pharmacodynamic models. Clin. Pharmacokin. 6:429453 (1981).

24. T. P. Green and B. L. Mirkin. Furosemide disposition in normal and proteinuric rats: urinary drug-protein binding as a determinant of drug excretion. J. Pharmacol. Exp. Ther. 218:122-127 (1981). 